\section{Standardising the clinical assessment of coronal knee laxity}

\author{
Jon V Clarke ${ }^{1,2}$, William T Wilson', Scott C Wearing ${ }^{3,4}$, \\ Frederic Picard', Philip E Riches ${ }^{2}$ and Angela H Deakin ${ }^{1,2}$
}

Proc IMechE Part $\mathrm{H}$ :

$\mathrm{J}$ Engineering in Medicine

226(9) 699-708

(c) IMechE 2012

Reprints and permissions:

sagepub.co.uk/journalsPermissions.nav DOI: $10.1177 / 09544$ II912451814

pih.sagepub.com

(SAGE

\begin{abstract}
Clinical laxity tests are used for assessing knee ligament injuries and for soft tissue balancing in total knee arthroplasty. This study reports the development and validation of a quantitative technique of assessing collateral knee laxity through accurate measurement of potential variables during routine clinical examination. The hypothesis was that standardisation of a clinical stress test would result in a repeatable range of laxity measurements.

Non-invasive infrared tracking technology with kinematic registration of joint centres gave real-time measurement of both coronal and sagittal mechanical tibiofemoral alignment. Knee flexion, moment arm and magnitude of the applied force were all measured and standardised. Three clinicians then performed six knee laxity examinations on a single volunteer using a target moment of $18 \mathrm{Nm}$.

Standardised laxity measurements had small standard deviations (within $1.1^{\circ}$ ) for each clinician and similar mean values between clinicians, with the valgus laxity assessment (mean of $3^{\circ}$ ) being slightly more consistent than varus (means of $4^{\circ}$ or $5^{\circ}$ ).

The manual technique of coronal knee laxity assessment was successfully quantified and standardised, leading to a narrow range of measurements (within the accuracy of the measurement system). Minimising the subjective variables of clinical examination could improve current knowledge of soft tissue knee behaviour.
\end{abstract}

\title{
Keywords
}

Knee laxity, clinical assessment, non-invasive assessment, ligament injuries, total knee arthroplasty

Date received: 19 December 20II; accepted: 16 May 2012

\section{Introduction}

Clinical laxity tests are frequently used for assessing knee ligament injuries and for soft tissue balancing in total knee arthroplasty (TKA). Current routine methods are subjective with respect to examination technique, magnitude of clinician-applied load and assessment of joint displacement. For collateral ligament injuries, scoring systems to grade severity are often based on millimetres of perceived joint opening with applied manual stress. ${ }^{1-3}$ The level of resolution required for this may exceed normal levels of human judgement and account for the frequent disparity between laxity examinations and true in-vivo joint function. ${ }^{4}$ Quantitative adjuncts, such as stress radiographs, have enabled a more objective measurement of joint space opening, but are still potentially limited by lack of standardisation of the applied load and are susceptible to limb positioning errors as a result of knee flexion or rotation. ${ }^{5,6}$ Soft tissues can be directly evaluated by magnetic resonance imaging (MRI), which is a commonly used imaging modality for diagnosis and grading of collateral ligament injuries. ${ }^{7-9}$ However, only static anatomical information is provided and so there is potential to underestimate the true extent of the injury. ${ }^{10}$

In TKA, assessment of laxity is a routine component of many soft tissue balancing techniques and is often used to determine the need for a soft tissue release, ${ }^{11,12}$ particularly for large deformities that are judged to be uncorrectable. Attempts have been made to categorise collateral laxity, for example Krackow's classification

\footnotetext{
'Department of Orthopaedics, Golden Jubilee National Hospital, UK ${ }^{2}$ Bioengineering Unit, University of Strathclyde, UK

${ }^{3}$ Faculty of Health Sciences and Medicine, Bond University, Australia ${ }^{4}$ Centre of Excellence for Applied Sport Science Research, Queensland Academy of Sport, Australia
}

\section{Corresponding author:}

Angela H Deakin, Department of Orthopaedics, Golden Jubilee National Hospital, Clydebank, West Dunbartonshire G8I 4DY, Scotland, UK. Email: angela.deakin@gjnh.scot.nhs.uk 
of medial ligament tightness, ${ }^{13}$ but this assumes that all clinicians have similar examination methods and are able to reliably judge knee alignment. However, human assessment of angles is known to be poor ${ }^{14}$ and the accuracy of alignment estimates under these circumstances may be no better than the order of $\pm 5^{\circ} .{ }^{15}$ Lower limb alignment measurements generated in real time, in the operating theatre by computer-assisted technology, have led to the development of quantitative TKA soft tissue balancing algorithms that are often based on tibiofemoral angular displacements with applied varus and valgus stress. ${ }^{16-18}$ However these techniques involve an unquantified manual load being applied by each individual surgeon, which may explain the difference in the derived values of the varus and valgus stress angles between studies. ${ }^{19}$ These assessments are also carried out on anaesthetised patients, so measured laxity may not be the same as the functional laxity of the knee under load. Furthermore, this technology relies on invasive bony tracker attachment and is, therefore, not currently available in a clinic setting.

Experimental models involving cadaveric knees ${ }^{20}$ or volunteer test rigs $^{21,22}$ have sought to standardise applied knee moments and measure the resultant angular displacements, but none have been successfully implemented into routine clinical practice. Therefore, despite the limitations of current techniques, manual knee laxity examination remains the primary means of diagnosing ligament injuries and assessing soft tissues in TKA. However with the high incidence of soft tissue knee injuries ${ }^{23,24}$ and the growing physical demands of TKA patients, ${ }^{25}$ there is a need to improve the evaluation of knee laxity to ensure better execution of corrective surgery.

The development of a quantitative assessment technique of coronal knee laxity for incorporation into current routine practice requires accurate standardisation of several parameters. The knee flexion angle should be determined and then maintained during the testing to minimise the potential positional variation in ligament restraining properties. ${ }^{4,15,26}$ The moment applied to the knee joint should be measured, which requires the identification of the force vector and moment arm of the applied load. The resultant displacement of tibia with respect to the femur should be quantified as a measure of laxity.

This study reports the development and validation of a quantitative technique of assessing collateral knee laxity through accurate measurement of potential variables during routine clinical examination. The hypothesis was that standardisation of a clinical stress test would result in repeatable range of laxity measurements.

\section{Materials and methods}

University ethical approval was granted for the healthy volunteer assessments.

\section{Knee flexion}

Non-invasive infrared (IR) tracking technology, with kinematic registration of joint centres to give mechanical lower limb alignment, ${ }^{27}$ was utilised for the real-time measurement of both coronal and sagittal mechanical tibiofemoral alignment. This system had been validated for coronal alignment ${ }^{27}$ but to verify this technology for recording flexion, comparison was made with a validated flexible electrogoniometer (EG) (Biometrics Ltd, Cwmfelinfach, Gwent, UK). ${ }^{28}$ A single volunteer was used for simultaneous measurements of both systems. Using the right lower limb, the EG was aligned along the estimated neutral mechanical axis of the hip-kneeankle joint centres, with the lower limb in full extension. The IR trackers were then attached over the top of the EG end plates (Figure 1). Once the lower limb was registered by the IR tracking system the knee was positioned and recorded in $0^{\circ}$ of flexion according to its onscreen display. This provided the 'zero' point for the EG, with synchronisation of the two systems performed at the start of each trial.

The knee was then passively flexed and held as stable as possible in $1^{\circ}$ increments, as indicated by the IR tracking system, up to $10^{\circ}$, and the precise angle at each point registered simultaneously by each system. The knee was then flexed in $10^{\circ}$ increments from $10^{\circ}$ to $100^{\circ}$. The trial was performed three times with the EG zeroed at the start of each set of measurements.

\section{Moment arm}

During laxity assessment, the moment arm is determined by the position of the clinician's hands. For this study, the controlled position of the manual force application was directly over the medial (valgus) or lateral (varus) ankle malleolus with the supporting hand placed over the medial (varus) or lateral (valgus) femoral epicondyle. The direction of the application of the force was assumed to be in the coronal plane and perpendicular to the mechanical axis of the tibia. Thus, the moment arm was the distance from the ankle centre

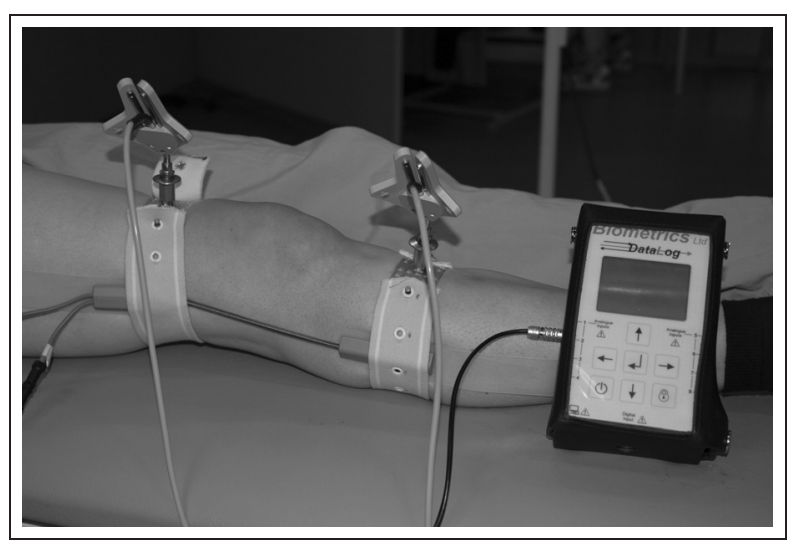

Figure I. Simultaneous attachment of flexible EG and noninvasive IR trackers to volunteer lower limb. 
to the knee centre; this distance could be determined from the non-invasive IR tracking system as the kinematic registrations identified the three-dimensional location of the knee and ankle centres. To validate the repeatability of the system for knee centre to ankle centre measurement, 20 separate registrations were performed by a single clinician on both a leg model with rigidly fixed tracker mounting pins and the right lower limb of a female volunteer (age 37, body mass index (BMI) 19), with the IR trackers removed and reapplied each time. To further assess repeatability, the same clinician carried out repeated measurements on 29 (18 male, 11 female) healthy volunteers of mean age 42 years (20-65) and mean BMI of 26 (19-34).

\section{Applied force}

A customised hand-held force application device (FAD) was incorporated into routine clinical knee examination, the geometry and dimensions of which were designed for ease of manual use. Two six degree of freedom Nano-25 force and torque sensors (ATI Industrial Automation, Apex, NC, USA) were positioned orthogonally on an external right-angled shell made out of $3 \mathrm{~mm}$ aluminium. The inner patientcontact surface was lined with expanded cross-linked polyurethane foam (Pe-Lite Medium - $5 \mathrm{~mm}$ thickness) secured to rectangular plates of $3 \mathrm{~mm}$ aluminium, which were attached to the tool adapter surfaces of the transducers. This internal aspect was designed to accommodate the ankle region with one contact surface acting as a leg support and the other applied to either the medial or lateral malleolus during force application (Figure 2).

After verifying the manufacturer's calibration, the two transducers were connected via an analogue to digital data acquisition (DAQ) system (NI USB-6229 M Series, National Instruments, Austin TX, USA) to a personal computer. LabView software (National Instruments, Austin TX, USA) was used to create a graphical user interface (GUI) to display the force data. During the clinical application of the FAD it was

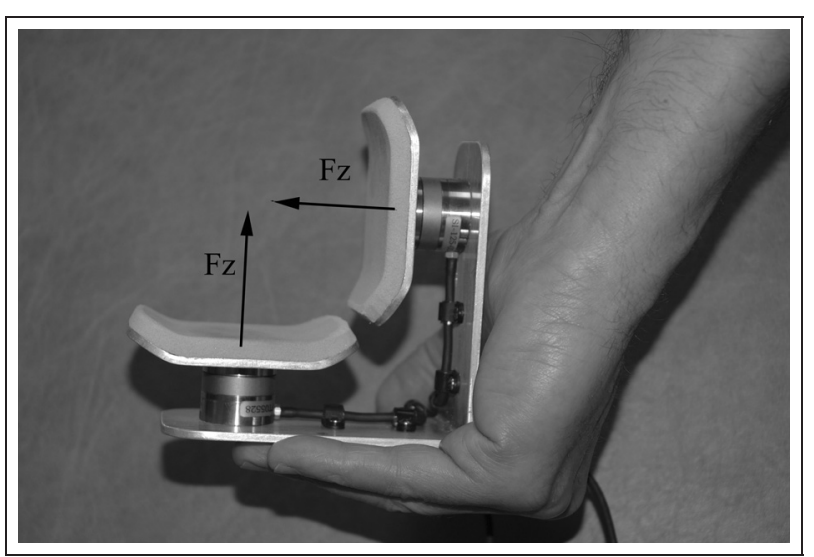

Figure 2. Hand-held force application device. assumed that most of the compressive loading owing to the varus and valgus stress manoeuvres would be along the $z$-axis of the horizontally aligned transducer, with the other supporting transducer measuring the force required to maintain the vertical leg position in addition to any hip flexor activity. To account for minor variations in the orientation of the FAD the GUI was configured to display the resultant force acting on each transducer. Only the resultant force acting on the horizontally aligned transducer was used to determine the applied moment.

To calibrate the FAD, incremental compressive loading, nominally along the $z$-axis of each transducer to represent the expected orientation in use, was applied. Loading from 0 to $40 \mathrm{~N}$ in $5 \mathrm{~N}$ steps was performed using an Instron 5800R uniaxial testing machine (Instron ${ }^{\circledR}$ Ltd, High Wycombe, Bucks, UK) fitted with a $100 \mathrm{~N}$ load cell (accuracy $0.1 \%$ full scale, $0.1 \mathrm{~N})$. The rate of loading was $10 \mathrm{~N} / \mathrm{s}$ and the load was maintained for $10 \mathrm{~s}$ for each increment. The Instron force data were recorded simultaneously via the DAQ to allow comparisons with the transducer force outputs.

\section{Measurement of applied moment}

Following initial validation of the FAD, the GUI displayed in real-time the applied force, subject-specific lever arm (from IR system) and the calculated resultant moment. The FAD was then used as a measuring device to determine the magnitude of the moments applied during routine clinical examination and so set a target limit for the FAD as a control device. Two clinicians (a consultant and a trainee orthopaedic surgeon) were instructed to perform 10 varus and valgus stress manoeuvres on the right knees of two volunteers (female aged 37 of BMI 19, male aged 40 of BMI 27) using the standardised manual positioning previously described. The FAD was held in the right palm during the application of clinically judged maximum varus and valgus loads with the knee in extension. The clinicians were blinded to the moment reading.

\section{Control of applied moment}

The GUI was further modified to display the moment as an ascending bar with the option of selecting a colour change as it reached pre-determined limits. An 'approaching target' and an 'at target' limit were incorporated (Figure 3). To further supplement this visual warning, an intermittent auditory signal was programmed to sound at the same limits and this allowed the examiner the option of remaining visually focussed on the examination technique.

Three clinicians (two consultant orthopaedic surgeons and one trainee orthopaedic surgeon) were then instructed to perform six varus and valgus knee laxity examinations on a single volunteer (female aged 37 of BMI 19) with the aim of applying a consistent moment 


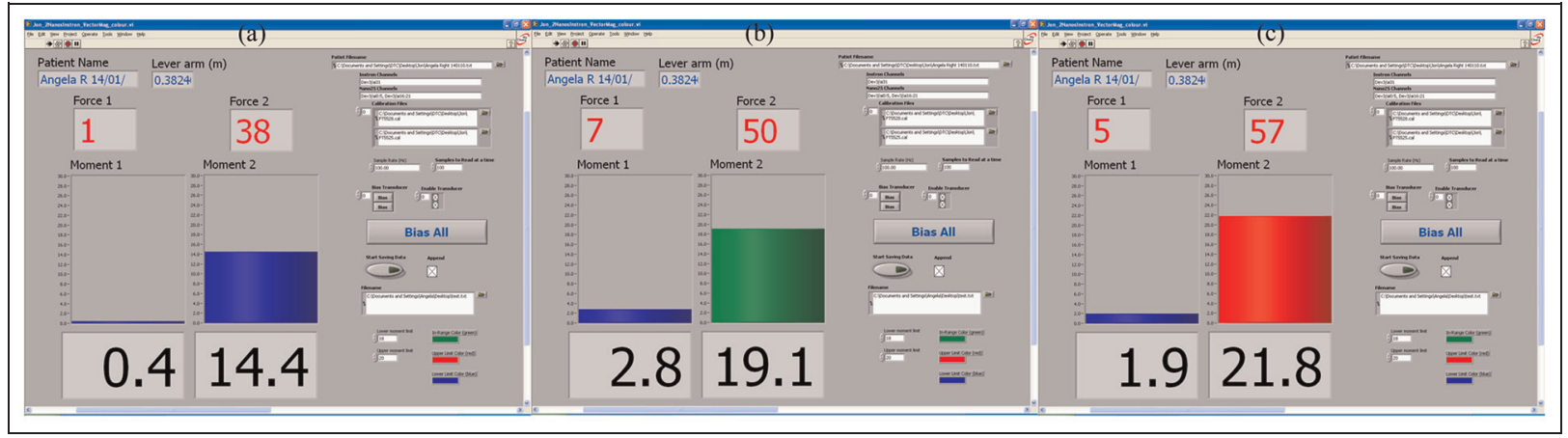

Figure 3. Customised LabView graphical displays illustrating the ascending bar for a chosen target of $20 \mathrm{Nm}$ with 'approaching' limit of $18 \mathrm{Nm}$ : (a) moment below first threshold; (b) moment above first threshold but below second; and (c) moment above second threshold.

of $18 \mathrm{Nm}$ as indicated by the FAD. An 'approaching' limit of $16 \mathrm{Nm}$ was selected. The technique was standardised as before but with an additional aim of maintaining the knee between $0^{\circ}$ and $5^{\circ}$ of flexion (target of $2^{\circ}$ ). The knee flexion was displayed in real time by the IR system. The clinicians were blinded to the corresponding laxity measurements for each applied moment. The IR system automatically recorded the maximum laxity angle achieved during each manoeuvre.

\section{Statistics}

Analysis was completed using Excel 2007 (Microsoft Corp, Redmond, WA, USA) and SPSS 17.0 (IBM Corp, Somers, NY, USA). Agreement between measurements (different systems or paired repeated data) was assessed using Bland-Altman plots with mean difference and limits of agreement for the difference calculated. ${ }^{29}$ Data were assessed for normality using the Korlmogorov-Smirnov (K-S) test. Comparison of variance between groups was made using the $\mathrm{F}$ Test with $p<0.05$ considered statistically significant. Correlation was assessed using Pearson's correlation coefficient.

To summarise results for non-parametric data median and range were used, whereas for parametric data mean and standard deviation (SD) were used.

\section{Results}

\section{Knee flexion}

The mean difference between the flexible EG and noninvasive IR tracking system for incremental angles of $1^{\circ}$ of knee flexion was $-0.2^{\circ}$ (limits of agreement for difference $\pm 0.8^{\circ}$ ) over all three trials (Figure 4(a)). For flexion to $100^{\circ}$, the mean difference was $-0.4^{\circ}$ (limits of agreement for difference $\pm 1.5^{\circ}$ ) over the three trials (Figure 4(b)). The discrepancy of the two systems was within $\pm 1^{\circ}$ for the majority of measurements.

\section{Moment arm}

Repeated moment arm calculations using the leg model produced normally distributed data $(p=0.934)$ with a $\mathrm{SD}$ of $1.5 \mathrm{~mm}$, while on a single volunteer the data were also normally distributed $(p=0.986)$, but SD was $4.8 \mathrm{~mm}$. This difference of variance was statistically significant $(p<0.001)$. For the paired volunteer measurements, the mean and limits of agreement for the difference was $1.5 \pm 13 \mathrm{~mm}$, with the Bland-Altman plot (Figure 5) illustrating that most agreed to within $\pm 10 \mathrm{~mm}$ ( $\leqslant 3 \%$ of leg length).

\section{Applied force}

The output signals from the FAD closely correlated to those of the Instron testing machine $(\mathrm{r}=0.999, p$ $<0.001$ ), although the traces were noisier (possibly owing to a poor earth connection). A typical trace is shown in Figure 6.

\section{Measurement of applied moment}

The mean overall applied moment, as chosen by the clinicians, was $22 \mathrm{Nm}$ (range 13-33) (Table 1). The mean moment applied to the knee of volunteer 1 was $19 \mathrm{Nm}$ (range 12-32), and based on this result the standardised moment to be applied during subsequent varus and valgus stress testing was $18 \mathrm{Nm}$.

\section{Control of applied moment}

The measured results for three clinicians during application of a target $18 \mathrm{Nm}$ moment are shown in Table 2. The overshoot ranged from 0 to $3.5 \mathrm{Nm}$, with an overall mean of $1.3 \mathrm{Nm}$. The laxity measurements showed small SD for each clinician and similar mean values between clinicians, with the valgus laxity assessment being slightly more consistent than varus (Table 2). 


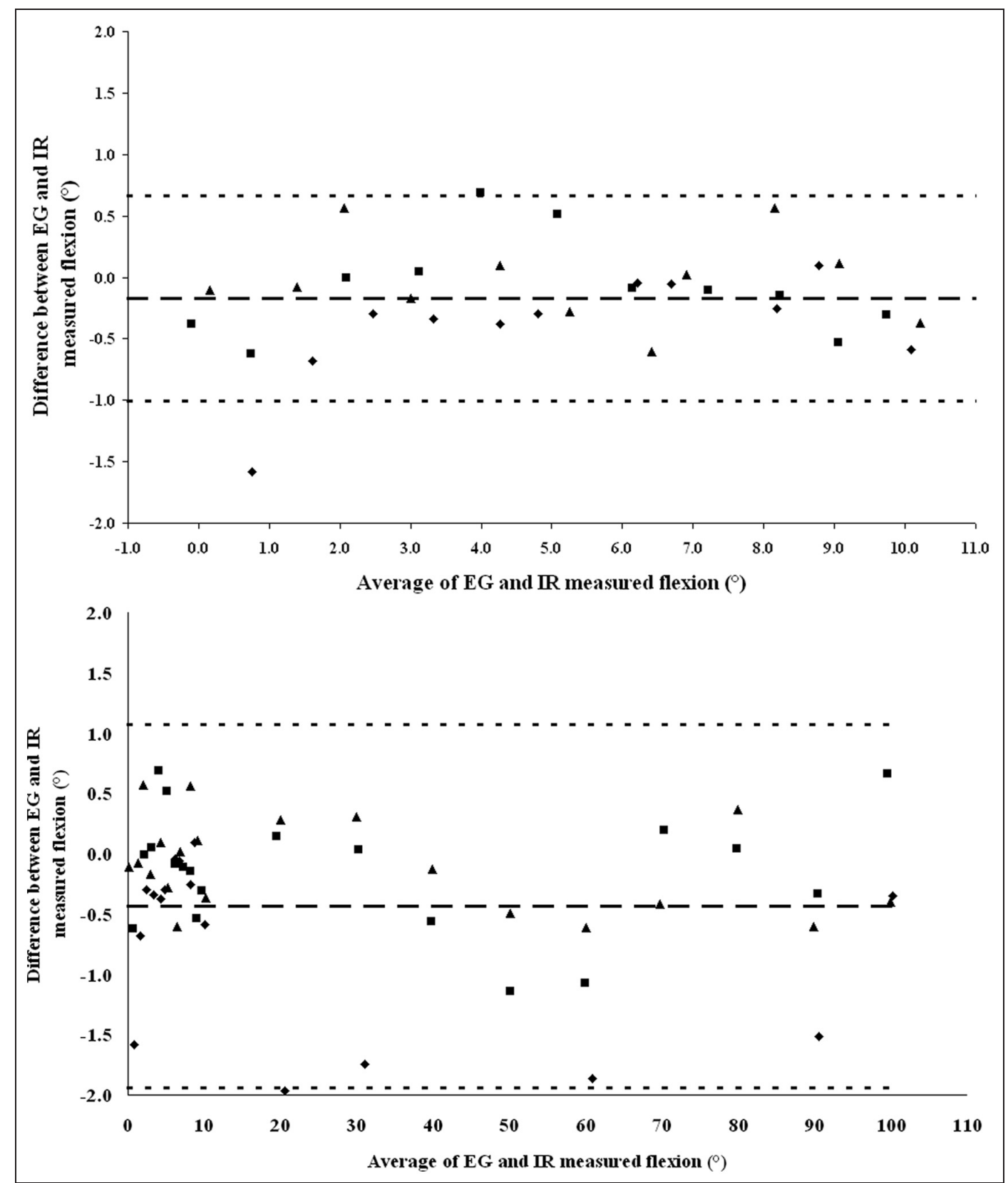

Figure 4. Bland-Altman plot showing limits of agreement for the EG and IR systems measuring the same knee flexion. Symbols indicate different trials ( trial I, $\square$ trial 2, $\boldsymbol{\Delta}$ trial 3). Long dash line is mean difference and small dashed lines give limits of agreement.

EG: electrogoniometer; IR: infrared.

\section{Discussion}

The assessment of collateral knee laxity by application of varus and valgus stress is an important clinical manoeuvre for evaluating ligament injuries and a fundamental component of many TKA soft tissue management techniques. This study aimed to overcome the subjective nature of current routine methods of assessment and develop a repeatable, objective stress test for incorporation into standard clinical practice. To achieve this, the accurate measurement and control of positional variables that could impact on the moment applied to the knee was required.
It was important to measure and maintain the flexion angle of the knee, as this determines the orientation and therefore the restraining properties of the collateral ligaments. ${ }^{15,26}$ The non-invasive IR technology used in this study provided a real-time display of sagittal alignment with measurements agreeing to within $1^{\circ}$ of those obtained with a validated flexible EG. ${ }^{28}$ This level of precision is far greater than human estimates of knee flexion, where levels of intra-observer variation can reach $20^{\circ}{ }^{30}$ In addition to its accuracy, the IR system could define the true position of knee flexion in comparison with the flexible EG, which would normally 


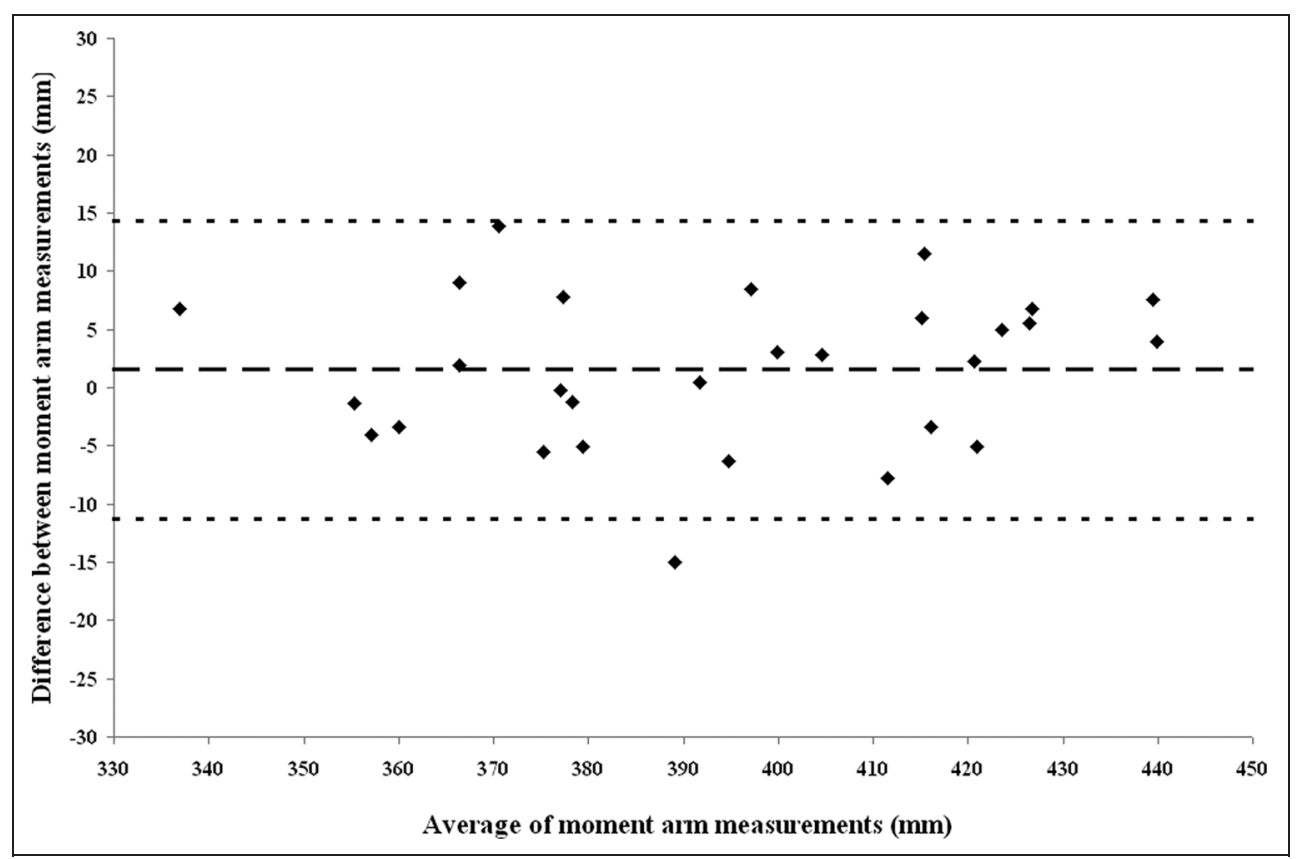

Figure 5. Bland-Altman plot showing limits of agreement between paired volunteer measurements of the effective moment arm. Long dash line is mean difference and small dashed lines give limits of agreement.

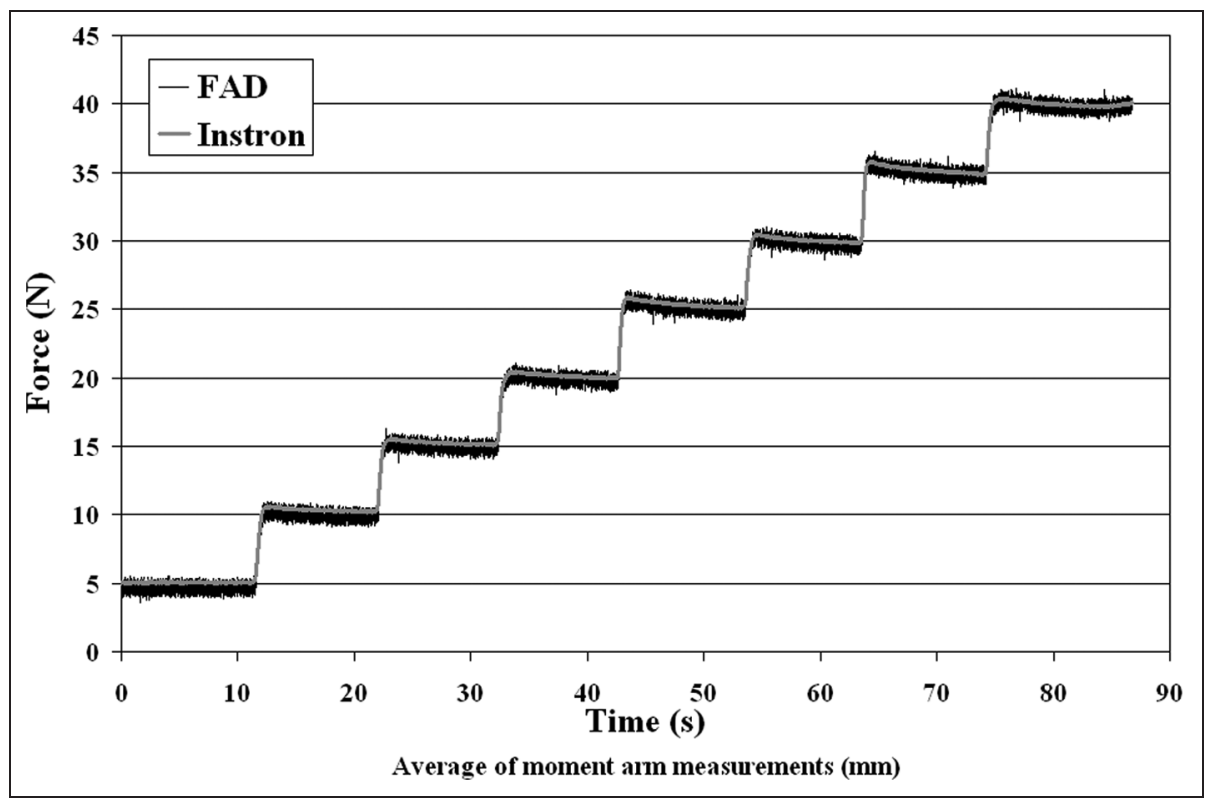

Figure 6. Simultaneous force output trace from the FAD (black) and Instron (grey) for $5 \mathrm{Nm}$ incremental compressive loads up to $40 \mathrm{Nm}$.

FAD: force application device.

Table I. Mean and range of moments recorded by FAD for repeated volunteer measurements.

\begin{tabular}{|c|c|c|c|c|}
\hline \multirow[t]{3}{*}{ Volunteer } & \multicolumn{4}{|c|}{ Applied moment $(\mathrm{Nm})$ mean [range] } \\
\hline & \multicolumn{2}{|l|}{ Clinician I } & \multicolumn{2}{|l|}{ Clinician 2} \\
\hline & Varus $(n=10)$ & Valgus $(n=10)$ & Varus $(n=10)$ & Valgus $(n=10)$ \\
\hline I & 20 [18-23] & $15[12-17]$ & 27 [24-32] & $16[|3-2|]$ \\
\hline 2 & 30 [27-33] & 19 [15-23] & 33 [29-36] & 20 [17-24] \\
\hline
\end{tabular}


Table 2. Mean and range of moments (recorded by the FAD) and corresponding angular displacements (measured by IR system) for repeated laxity tests on a single volunteer.

\begin{tabular}{|c|c|c|c|c|}
\hline \multirow[t]{2}{*}{ Clinician } & \multicolumn{2}{|c|}{ Mean moment [range] $(\mathrm{Nm})$} & \multicolumn{2}{|c|}{ Mean laxity \pm SD $\left(^{\circ}\right)$} \\
\hline & Varus $(n=6)$ & Valgus $(n=6)$ & Varus $(n=6)$ & Valgus $(n=6)$ \\
\hline I & 19 [18.8-19.9] & $19[18.3-20.1]$ & $5 \pm 1.1$ & $3 \pm 0.2$ \\
\hline 2 & $20[18.0-2 \mid .5]$ & $20[19.1-20.3]$ & $4 \pm 0.8$ & $3 \pm 0.7$ \\
\hline 3 & $19[18.4-19.1]$ & $19[18.3-19.1]$ & $5 \pm 0.3$ & $3 \pm 0.4$ \\
\hline
\end{tabular}

rely on an initial estimate of $0^{\circ}$ as its starting point. One limitation of this study is that the flexible EG is also sensitive to soft tissue movements, i.e. it does not quantify actual bone motion. However, given the use of healthy volunteers, it was not possible to use an invasive method of tracking of the actual bone motions. The flexible EG is a widely accepted non-invasive technique for quantifying knee flexion.

With control of knee flexion it was then necessary to standardise the clinical examination technique in order to define a moment arm. The moment arm was defined as the distance between the knee and ankle centres as calculated by the IR system. To use this then required careful hand positioning according to the surface anatomy, so that the force application coincided with the knee and ankle centres. For the measurement of the moment arm distance, repeated measurements on a single volunteer $(\mathrm{SD} \pm 4.8 \mathrm{~mm})$ compared well with the variation owing to the system precision, as measured by a leg model with rigid tracker mountings $(\mathrm{SD} \pm 1.5 \mathrm{~mm})$. Although this difference was statistically significant, it represented only a small loss of accuracy from soft tissue artefacts. Further to this, the measurement of this distance was repeatable to $\pm 13 \mathrm{~mm}$ when performed on 29 volunteers. Therefore, the technique may be more accurate than currently available routine methods of leg length assessment, such as a measuring tape or radiographs. Assuming a moment arm of $0.4 \mathrm{~m}, \mathrm{a} \pm 13 \mathrm{~mm}$ change would equate to a $\pm 0.65 \mathrm{Nm}$ variation in moment. This is relatively small compared with the range of moments seen. In terms of the point of force application coinciding with this pre-defined distance, the surface anatomy allowed control of hand and FAD placement. However, a limitation of the study is that the position of the FAD was not known throughout the stress manoeuvre. This could be overcome by tracking of the FAD to give the precise point of force application and so calculate a 'true' lever arm throughout the laxity assessment manoeuvre.

To standardise the moment required the control of the applied force. Much of the previous work in this area has relied on invasive access to the knee through either the use of cadavers or intra-operative studies in the context of TKA, where gap-balancing devices have helped to quantify the amount of force application. Classic techniques have involved spacer blocks as a surrogate measure of soft tissue tension, ${ }^{31}$ or laminar spreaders that enabled a comparison between medial and lateral joint spaces both in flexion and extension. More sophisticated designs have incorporated scales to measure the amount of gap distraction ${ }^{32,33}$ or force sensing devices to determine the applied load. D'Lima et al. ${ }^{34,35}$ utilised a force transducer to measure intraarticular compressive forces on the tibial component of knee replacements and a similar device that measured knee joint moments and forces was developed and evaluated in-vitro by Crottet et al. ${ }^{36}$ The use of cadaveric specimens has permitted direct attachment of strain gauges to bone with accurate measurement of the applied moment-load. Methods of applying standardised varus-valgus moments to the knee joint have ranged from basic weight-pulley systems ${ }^{15,22}$ to digital strain gauges. $^{20,37}$

Most in-vivo studies that have sought to standardise varus and valgus loads to the knee have involved cumbersome experimental set-ups that are not readily adaptable to a clinical setting. Sharma et al. ${ }^{21}$ developed a bench with an attached low-friction track to support the leg and a hand-held dynamometer to apply a fixed load. Van der Esch et al. ${ }^{22}$ constructed a measurement chair, with a lower limb attachment consisting of five specific fixation points relative to the knee joint line, an electronic meter to record angular deviation and a weight-pulley system to deliver a standardised load. In addition to the impracticalities of these set-ups, the laxity measurements were of limited use in clinical practice owing to considerable intra- and inter-observer measurement error. From the point of view of clinicianapplied varus-valgus loads however, any measuring device should consider the way in which patients are examined. The FAD designed in this study allowed the incorporation of commercial transducers without affecting the magnitude of their force measurements. In particular, the deformation of internal patient contact surface (polyurethane pad) did not result in a measureable change in the transmitted loads. The design allowed the FAD to be incorporated into a routine clinical assessment with minimal alteration of examination technique. While we are not aware of any similar devices for measuring collateral knee laxity, there are reports of measurement tools for recording manual contact forces. Van Zoest et al. ${ }^{38}$ recognised the importance of manual techniques in disciplines, such as chiropractic and osteopathy, and developed a similar palm-held force measurement system for improving the 
manual perception and force delivering skills of student practitioners. Harms and Bader $^{39}$ investigated the variability of forces applied by therapists during spinal mobilisation procedures through the construction of an instrumented mobilisation coach that could measure the magnitude and direction of applied forces. In spite of standardising the manipulation technique, there was a large variation in the forces used by different therapists, ranging from 63 to $347 \mathrm{~N}$. In comparison, the varus and valgus knee moments recorded by the FAD in this study were more consistent for the two clinicians assessed, with a range of measurements from 13 to $33 \mathrm{Nm}$ for a total of 80 stress manoeuvres. This may reflect a more perceptible endpoint for the constraining soft tissues of the knee, ${ }^{15}$ in contrast to the underlying tissues around the spine that may not provide obvious feedback when applying a manual compressive force. The mean recorded moment of $19 \mathrm{Nm}$ did not produce any discomfort in the particular individual tested, despite being higher than the $12 \mathrm{Nm}$ upper subjecttolerance limit that has often been used in other studies. $^{20-22}$ This may be due to the short duration of the stress manoeuvre compared with the more sustained loads in these experimental studies.

Following its use as a measurement tool, the FAD was utilised as a control device through the design of a GUI, which provided a repeatable method of applying a pre-determined moment. The visual and auditory warning systems were effective in preventing significant overshoot of the selected threshold, with a mean of $1.3 \mathrm{Nm}$. The range of manual forces moments when using the FAD $(3.5 \mathrm{Nm})$ was about half of those selected by clinicians $(8 \mathrm{Nm})$ for any given condition. This indicates that it can satisfactorily be used as a control device.

For each applied varus and valgus load, the corresponding coronal angular displacement of the knee, from its resting position, was used to quantify laxity. The repeated sets of measurements by three clinicians had similar mean values, with the SD ranging from $\pm .2^{\circ}$ to $\pm .1^{\circ}$. The small differences between clinicians may be due to actual variations in the applied moment, but may also result from the $\pm .1^{\circ}$ accuracy of the noninvasive IR tracking technology. However, this represents a significantly higher degree of precision than the likely $\pm 5^{\circ}$ human error in estimation of alignment. ${ }^{15}$ The system also has a number of potential advantages over alternative non-invasive laxity assessments. The radiographic measurement of joint space opening has been widely reported, $3,20,40$ but drawbacks include the use of ionising radiation and the requirement for meticulous control of lower limb positioning. The grading of collateral ligament injury severity on the basis of a $1-2 \mathrm{~mm}$ difference in gap opening ${ }^{20}$ could potentially be seen with small rotational and sagittal variations in knee position. ${ }^{6,13}$ An alternative approach to measuring laxity involves the use of specially designed mechanical devices incorporating goniometers. Early cadaveric work was limited by inaccurate manual measurement tools to record the resultant displacement following an applied load. ${ }^{41}$ Markolf et al. ${ }^{15}$ addressed these limitations by using a specially designed three-dimensional goniometer linkage that allowed the knee joint to be maintained at a specific degree of flexion, while electronically recording the resultant varus-valgus angulation. Unfortunately, in-vivo adaptation of goniometers has generally involved cumbersome experimental set-ups which, similar to previously described force application technology, are not practical for routine clinical use.

Although some of the disadvantages of other systems have been addressed, the measurement tool developed in this study also has limitations. For the manually applied force, it was assumed that the loading of the transducers within the FAD was perpendicular to the tibial mechanical axis in the coronal plane. However, in spite of careful positioning of the device, the true orientation of the resultant force vector was unknown. Using the IR tracking system to give the real-time orientation of the FAD relative to the defined tibial moment arm could potentially overcome this. During laxity testing, although the auditory warning system enabled clinicians to remain more focussed on examination technique, there was still the requirement to use the on-screen display of flexion to control knee position. The use of a device to hold the knee in a specified degree of flexion, such as a wedge in the popliteal fossa or the use of an image overlay, ${ }^{42}$ could remove the requirement to look at a computer screen during clinical examination.

A further limitation of this study is that the final assessment was carried out on one slim volunteer with no 'true' measurement of laxity. As stated above, it was not possible to use an invasive method of assessment on this healthy volunteer. Also, it was not possible to image the knee under stress. However, in terms of the accuracy of the IR system on larger individuals, it has been shown to be highly repeatable on a group of subjects of BMI up to 34 , with clinical examination not affecting alignment measurements. ${ }^{27}$ This had been taken to indicate that the tracker mountings are very stable. The additions to the assessment, including the use of the FAD, should not have affected the accuracy of the IR system in any way, so it is felt that the previous results give confidence that this set-up would work with the higher BMI population seen for total knee replacements. Further to this, the authors intend to carry out more work to use this set-up in a patient population with osteoarthritis due to undergo TKA and to compare non-invasive measurements directly with invasive intra-operative data. However, it is necessary to validate the system on healthy volunteers before proceeding to this step.

In spite of the potential limitations of our system, we have successfully quantified and standardised the manual technique of coronal knee laxity assessment for the limited number of subjects and clinicians evaluated. Our hypothesis stated that this would lead to a narrow range of laxity measurements, which we have confirmed as the results were within the accuracy limits of our IR 
system. Minimising the subjective variables of clinical examination with a more repeatable, quantitative technique could improve current knowledge of soft tissue knee behaviour. This may lead to improved balancing techniques in TKA through quantification of knee laxity before, during and after surgery, enabling a more widespread use of single surgeon-derived algorithms. ${ }^{16,17,18}$ There is a potential role in the management of collateral ligament injuries with regard to more reliable initial diagnosis and severity grading, as well as monitoring recovery and rehabilitation. Standardised data from healthy, injured and osteoarthritic knees could improve our knowledge of normal and abnormal knee kinematics, and lead to more objective treatment algorithms. Finally, as this augmented learning can be incorporated into traditional examination techniques, the ability to quantify the technique of senior clinicians may help to enhance the perceptive skills of more junior trainees who do not have the benefit of experience.

\section{Funding}

This research received no specific grant from any funding agency in the public, commercial, or not-for-profit sectors. However the Department of Orthopaedics, Golden Jubilee National Hospital has received research funding from BBraun Aesculap and a proportion of this has been used to fund a fixed term research post for one of the authors (JVC).

\section{Acknowledgements}

The authors would like to thank Mr Stephen Murray for assistance with the design and manufacture of the straps, Mr David Smith and Mr Stephen Murray for their technical assistance with the design, development and testing of the FAD and Mr Hin Lau for his provision of the LabView code to collect data from the nanotransducers. They would also like to thank BBraun Aesculap for the loan of equipment used in this project.

\section{Conflict of interest}

One of the authors (FP) has patents/licensing agreements with BBraun Aesculap. The other authors (JVC, WTW, SCW, PER and AHD) certify that they have no commercial associations (e.g. consultancies, stock ownership, equity interest, patent/licensing arrangements, etc.) that might pose a conflict of interest in connection with the submitted article.

\section{References}

1. Petermann $\mathbf{J}$ and von Garrel, Gotzen. Non-operative treatment of acute medial collateral ligament lesions of the knee joint. Knee Surg Sports Traumatol Arthrosc 1993; 1: 93-96.

2. Hefti F, Müller W, Jakob RP, et al. Evaluation of knee ligament injuries with the IKDC form. Knee Surg Sports Traumatol Arthrosc 1993; 1: 226-234.
3. Wijdicks CA, Griffith CJ, Johansen S, et al. Injuries to the medial collateral ligament and associated medial structures of the knee. J Bone Joint Surg Am 2010; 92-A: 1266-1280.

4. Noyes FR, Grood ES, Butler DL, et al. Knee ligament tests: what do they really mean? Physical Therapy 1980; 60: 1578-1581.

5. Krackow KA, Pepe CL and Galloway EJA. A mathematical analysis of the effect of flexion and rotation on apparent varus/valgus alignment at the knee. Orthopaedics 1990; 13: 861-868.

6. Siu D, Cooke TD, Broekhoven LD, et al. A standardised technique for lower limb radiography. Practice, applications, and error analysis. Invest Radiol 1991; 26: 71-77.

7. Rasenberg EI, Lemmens JA, van Kampen A, et al. Grading medial collateral ligament injury: comparison of MR imaging and instrumented valgus-varus laxity testdevice. A prospective double-blind patient study. Eur $J$ Radiol 1995; 21: 18-24.

8. Beall DP, Googe JD, Moss JT, et al. Magnetic resonance imaging of the collateral ligaments and the anatomic quadrants of the knee. Radiol Clin North Am 2007; 45: 983-1002.

9. Ikuma H, Abe N, Uchida Y, et al. Novel magnetic resonance imaging evaluation for valgus instability of the knee caused by medial collateral ligament injury. Acta Med Okayama 2008; 62: 185-191.

10. Jacobson KE and Chi FS. Evaluation and treatment of medial collateral ligament and medial-sided injuries of the knee. Sports Med Arthrosc 2006; 14: 58-66.

11. Whiteside LA. Soft tissue balancing. J Arthroplasty 2002; 17(suppl. 1): 67-72.

12. Mihalko WM, Whiteside LA and Krackow KA. Comparison of ligament-balancing techniques during total knee arthroplasty. J Bone Joint Surg Am 2003; 85A(Suppl. 4): 132-135.

13. Krackow KA. Varus deformity. In: The technique of total knee arthroplasty. St Louis, Missouri: CO Mosby Company, 1990, pp.317-340.

14. Edwards JZ, Greene KA, Davis RS, et al. Measuring flexion in knee arthroplasty patients. J Arthroplasty 2004; 19: 369-372.

15. Markolf KL, Mensch JS and Amstutz HC. Stiffness and laxity of the knee- the contributions of the supporting structures. J Bone Joint Surg Am 1976; 58-A: 583-594.

16. Saragaglia D, Chaussard C and Rubens-Duval B. Navigation as a predictor of tissue release during 90 cases of computer assisted total knee arthroplasty. Orthopaedics 2006; 29(10 Suppl): 137-139.

17. Picard F, Deakin AH, Clarke JV, et al. A quantitative method of effective soft tissue management for varus knees in total knee replacement surgery using navigational techniques. Proc IMechE Part H: J Engineering in Medicine 2007; 221: 763-772.

18. Hakki S, Coleman S, Saleh K, et al. Navigational predictors in determining the necessity for collateral ligament release in total knee replacement. $J$ Bone Joint Surg Br 2009; 91-B: 1178-1182.

19. Bellemans J, D'Hooghe P, Vandenneucker H, et al. Soft Tissue balance in total knee arthroplasty: does stress relaxation occur perioperatively? Clin Orthop Relat Res 2006; 452: 49-52.

20. LaPrade RF, Heikes C, Bakker AJ, et al. The reproducibility and repeatability of varus stress radiographs in the 
assessment of isolated fibular collateral ligament and grade-III posterolateral knee injuries. An in vitro biomechanical study. J Bone Joint Surg Am 2008; 90: 20692076.

21. Sharma L, Congron L, Felson DT, et al. Laxity in healthy and osteoarthritic knees. Arthritis Rheum 1999; 42: 861-870.

22. van der Esch M, Steultjens M, Ostelo RWJG, et al. Reproducibility of instrumented knee joint laxity measurement in healthy subjects. Rheumatology 2006; 45: 595-599.

23. Miyasaka KC, Daniel DM and Stone ML. The incidence of knee ligament injuries in the general population. Am $J$ Knee Surg 1991; 4: 43-48.

24. American Academy of Orthopaedic Surgeons. Common knee injuries. http://orthoinfo.aaos.org/topic.cfm?topic $=$ A00325 (2007, accessed 30 November 2010).

25. Nilsdotter AK, Toksvig-Larsen S and Roos EM. Knee arthroplasty: are patients' expectations fulfilled? A prospective study of pain and function in 102 patients with 5-year follow-up. Acta Orthop 2009; 80: 55-61.

26. Grood ES, Noyes FR, Butler DL, et al. Ligamentous and capsular restraints preventing straight medial and lateral laxity in intact human cadaver knees. J Bone Joint Surg Am 1981; 63: 1257-1269.

27. Clarke JV, Riches PE, Picard F, et al. A non-invasive system for the measurement of knee alignment. Comp Aided Surg. Comp Aided Surg 2012; 17: 29-39.

28. Rowe PJ, Myles CM, Hillmann SJ, et al. Validation of flexible electrogoniometry as a measure of joint kinematics. Physiotherapy 2001; 87: 479-488.

29. Bland JM and Altman DG. Statistical methods for assessing agreement between two methods of clinical measurement. Lancet 1986; 1: 307-310.

30. Cushnaghan J, Cooper C, Dieppe P, et al. Clinical assessment of osteoarthritis of the knee. Ann Rheum Dis 1990; 49: $768-770$
31. Insall JN, Binazzi R, Soudry $\mathrm{M}$, et al. Total knee arthroplasty. Clin Orthop 1985; 192: 13-22.

32. Unitt L, Sambatakakis A, Johnstone D, et al. Short-term outcome in total knee replacement after soft-tissue release and balancing. J Bone Joint Surg Br 2008; 90-B: 159-165.

33. Winemaker MJ. Perfect balance in total knee arthroplasty: the elusive compromise. J Arthroplasty 2002; 17: 2-10.

34. D'Lima DD, Townsend CP, Arms SW, et al. An implantable telemetry device to measure intra-articular tibial forces. J Biomech 2005; 38: 299-304.

35. D'Lima DD, Patil S, Steklov N, et al. Dynamic intraoperative ligament balancing for total knee arthroplasty. Clin Orthop Relat Res 2007; 463: 208-212.

36. Crottet D, Maeder T, Fritschy D, et al. Development of a force amplitude- and location-sensing device to improve the ligament balancing procedure in TKA. IEEE Trans Bio Eng 2005; 52: 1609-1611.

37. van Damme G, Defoort K, Ducoulombier Y, et al. What should the surgeon aim for when performing computerassisted total knee arthroplasty? J Bone Joint Surg Am 2005; 87-A(suppl. 2): 52-58.

38. van Zoest GGJM, van den Berg HTCM and Holtkamp FC. Three-dimensionality of contact forces during clinical manual examination and treatment: a new measuring system. Clin Biomech 2002; 17: 719-722.

39. Harms MC and Bader DL. Variability of forces applied by experienced therapists during spinal mobilization. Clin Biomech 1996; 12: 393-399.

40. Moore TM, Meyers MH and Harvey JP. Collateral ligament laxity of the knee. Long-term comparison between plateau fractures and normal. J Bone Joint Surg Am 1976; 58-A: 594-598.

41. Brantigan OC and Voshell AF. The mechanics of the ligaments and menisci of the knee joint. $J$ Bone Joint Surg 1941; 23: 44-66.

42. Moody JE, Nikou C, Picard F, et al. Computer-integrated anterior cruciate ligament reconstruction system. $J$ Bone Joint Surg Am 2002; 84: S99-S101. 